\title{
Primary Lymphoma of Internal Acoustic Meatus Mimicking Vestibular Schwannoma-A Rare Diagnostic Dilemma
}

\author{
Narayan Jayashankar ${ }^{1}$ Swati Kodur ${ }^{10}$ Deepak Patkar ${ }^{2}$ Mitusha Verma ${ }^{2}$ \\ ${ }^{1}$ Department of Otorhinolaryngology, Dr. Balabhai Nanavati Hospital, \\ Mumbai, Maharashtra, India \\ 2 Department of Radiology, Dr. Balabhai Nanavati Hospital, Mumbai, \\ Address for correspondence Swati Kodur, MBBS, DLO, Department of \\ ENT, Dr. Balabhai Nanavati Hospital, SV Road, Vile Parle West, \\ Maharashtra, India
}

J Neurol Surg Rep 2021;82:e1-e5.

\begin{abstract}
Background/Setting A subject presenting with a unilateral sensorineural hearing loss and with vertigo/imbalance and a lesion of internal acoustic meatus (IAM) most often represents a vestibular schwannoma. Several alternative pathologies involving the region, with clinical and neuroradiological similarities, could lead to an error in judgement and management. Rare tumors of the IAM pose unique diagnostic difficulty. A rare case that we present here had a typical history and imaging findings suggestive of vestibular schwannoma. A primary central nervous system (CNS)

Keywords

- vestibular schwannoma

- internal acoustic meatus

- CNS lymphoma

- imaging of internal acoustic meatus lesions

lymphoma was diagnosed in later stages of brain involvement warranting a retrospective analysis of the entity.

Case Summary An 80-year-old male presented with unilateral sensorineural hearing loss, vertigo, and imbalance. On imaging, he was found to have a lesion in the left internal auditory meatus, reported as a vestibular schwannoma and operated upon. Subject's condition worsened with time and a repeat imaging was suggestive of a CNS lymphoma with lesions involving bilateral cerebellum and subcortical white matrix.

Conclusion To conclude, primary CNS lymphoma presenting an isolated lesion in the IAM with no other parenchymal lesions at presentation is a rare incidence; to our knowledge this is the first case of such unique presentation.
\end{abstract}

\section{Introduction}

A subject presenting to the outpatient department with unilateral hearing loss, disequilibrium/giddiness, tinnitus, and a space-occupying lesion) in the internal acoustic meatus (IAM) on imaging usually diverts the clinicians toward suspecting a vestibular schwannoma (VS). Vestibular schwannoma represents 6 to $7 \%$ of all intracranial tumor entity with incidence of 1 in $100000^{1-5}$ with nonschwan- noma tumors being seldom diagnosed (accounting only 10\% of lesions). ${ }^{6-8}$

We, through this write up, aim at discussing pathologies that mimic VS clinically and neuroradiologically leading to an error in judgement and management. Rare tumors of the IAC include lipomas, hemangiomas, meningiomas, neurofibromas, hamartomas, and arachnoidal cysts with each posing unique diagnostic difficulties. ${ }^{7}$ A rare case that we present here had a classical history and imaging, convincing received

March 26, 2019

accepted after revision

February 25, 2020
DOI https://doi.org/

$10.1055 / \mathrm{s}-0040-1722343$. ISSN 2193-6358.

\footnotetext{
(C) 2021. The Author(s).

This is an open access article published by Thieme under the terms of the Creative Commons Attribution-NonDerivative-NonCommercial-License, permitting copying and reproduction so long as the original work is given appropriate credit. Contents may not be used for commercial purposes, or adapted, remixed, transformed or built upon. (https://creativecommons.org/ licenses/by-nc-nd/4.0/) Georg Thieme Verlag KG, Rüdigerstraße 14, 70469 Stuttgart, Germany
} 

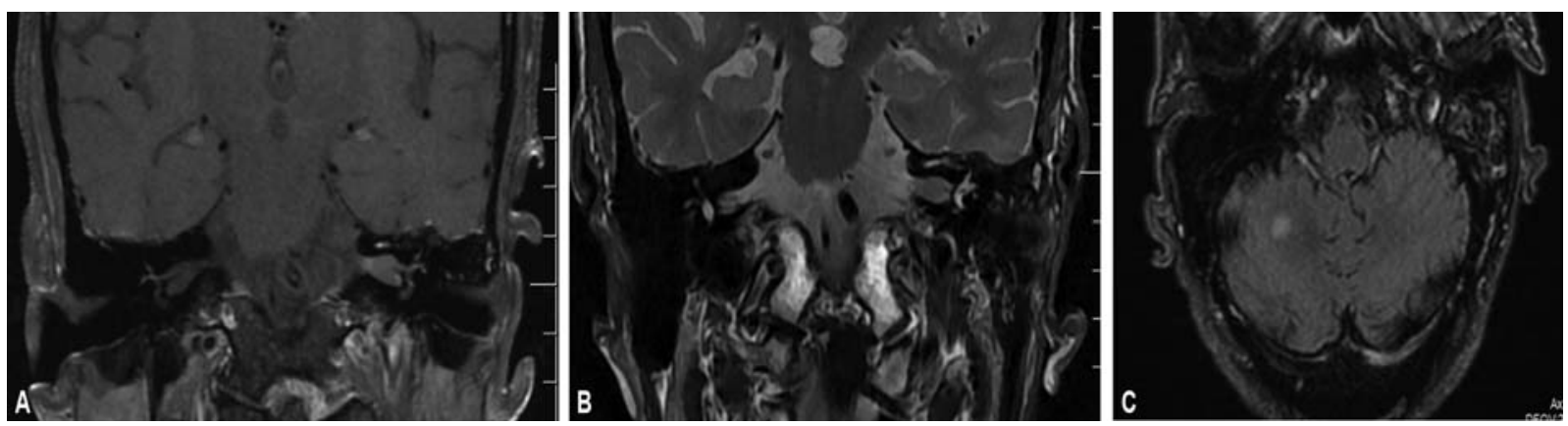

Fig. 1 (A, B) Coronal T1 postcontrast and coronal T2 images showing moderately enhancing lesion in the left internal auditory canal. (C) Small enhancing lesion in the right cerebral hemisphere. The lesion is fluid-attenuated inversion recovery hyperintense and hypoperfused.

us of a VS, with a primary central nervous system (CNS) lymphoma being diagnosed in later stages of brain involvement warranting a retrospective analysis of the entity.

\section{Case Report}

An 80-year-old male presented to our outpatient department with a history of progressively worsening hearing loss on the left ear, with vertigo and imbalance during walking, of 2 months duration. The symptoms were unnerving and hampering his daily activities. Pure tone audiometry was performed and indicated a severe-to-profound left-sided sensorineural hearing loss with preserved hearing on the right ear. Magnetic resonance imaging (MRI) scan with gadolinium was performed that showed a $1.5 \times 0.8 \times 0.5 \mathrm{~cm}$ lesion located in the intracanalicular portion of the left internal auditory meatus, isointense on $\mathrm{T} 1$ and hypointense on T2, with heterogeneity suggestive of a VS ( - Fig. 1A and B). High-resolution computed tomography (HRCT) temporal bone done preoperatively demonstrated a mild widening of the left IAM. In the same scan, there were small discoid enhancing lesions in both cerebellar hemispheres that were hyperintense on $\mathrm{T} 2$ and isointense on $\mathrm{T} 1$ sequences. They were considered as ischemic foci; a MR spectroscopy and perfusion was done that appeared hypoperfused on 3D arterial spin labeling (noncontrast perfusion) and dynamic susceptibility contrast perfusion and showed normal spectral pattern, suggestive of ischemic foci (- Fig. 1C).
Having, thus, obtained radiological confirmation of VS and a characteristic history supporting the diagnosis led us to suggest surgery to the subject due to his incapacitating symptomatology. A translabyrinthine approach was performed since there was profound hearing loss on the left ear. On opening the IAM, surprisingly, no lesion was detected within the meatus. The dura of the IAM was incised open and sent for histopathological examination that subsequently was reported as inflammatory. Meanwhile, the patient's complaints of vertigo and imbalance worsened with repeated falling that prompted a repeat MRI scanning of the brain within 3 weeks wherein new findings of fairly large, ill-defined lesions with altered signal intensity were identified in bilateral cerebellar hemispheres and left anterior subcortical white


A navigation biopsy of the lesion, later, suggested a nonHodgkin's lymphoma. Serial scans of the subject through 1-year follow-up are as depicted in - Fig. 3A-D.

A positron emission tomography scan was done that negated any other system involvement and a diagnosis of primary CNS lymphoma was made ( - Fig. 4). Patient underwent chemotherapy for the same. Warranting a retrospective analysis was the fact that the patient presented with a history and imaging mimicking VS, adding much to clinician's dilemma.

Diffusion-weighted imaging (DWI) sequence is a part of all our standard MRI protocols and was performed in this case as well. No area of altered diffusivity was identified on DWI images in this particular patient (-Fig. 5A, B).
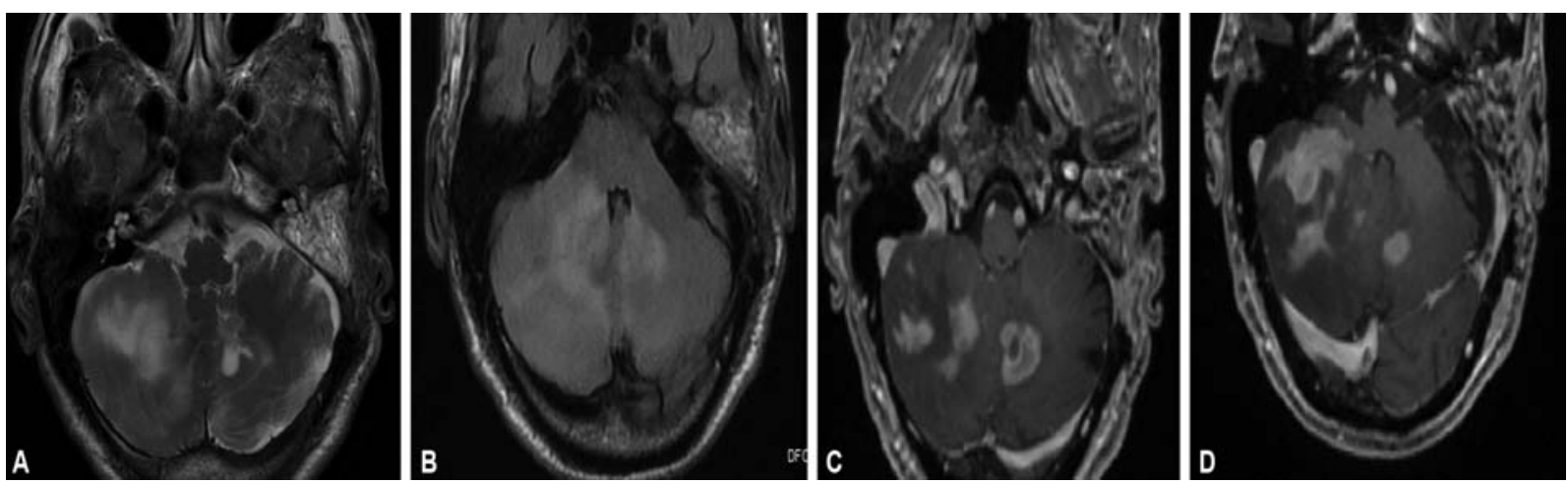

Fig. 2 (A) Axial T2-weighted imaging showing patchy area of altered signal intensity scattered in bilateral cerebellar hemisphere. (B) Axial fluidattenuated inversion recovery images showing area of hyperintensities in cerebellar hemisphere. (C, D) On axial T1-weighted images, these areas of altered signal intensity show moderate heterogenous enhancement. 

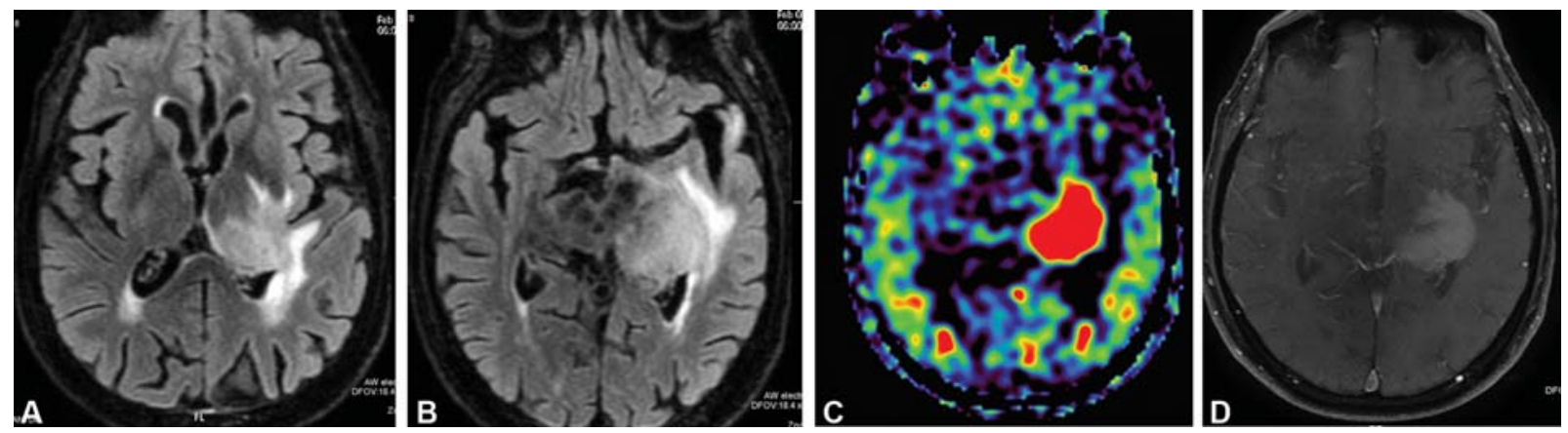

Fig. 3 (A, B) Axial fluid-attenuated inversion recovery images showing area of altered signal intensity in left thalamus and basal ganglia region. (C) Axial arterial spin labeling image showing hyperperfusion in these regions of altered signal intensity. (D) On axial T1-weighted images, these areas of altered signal intensity show moderate heterogenous enhancement.
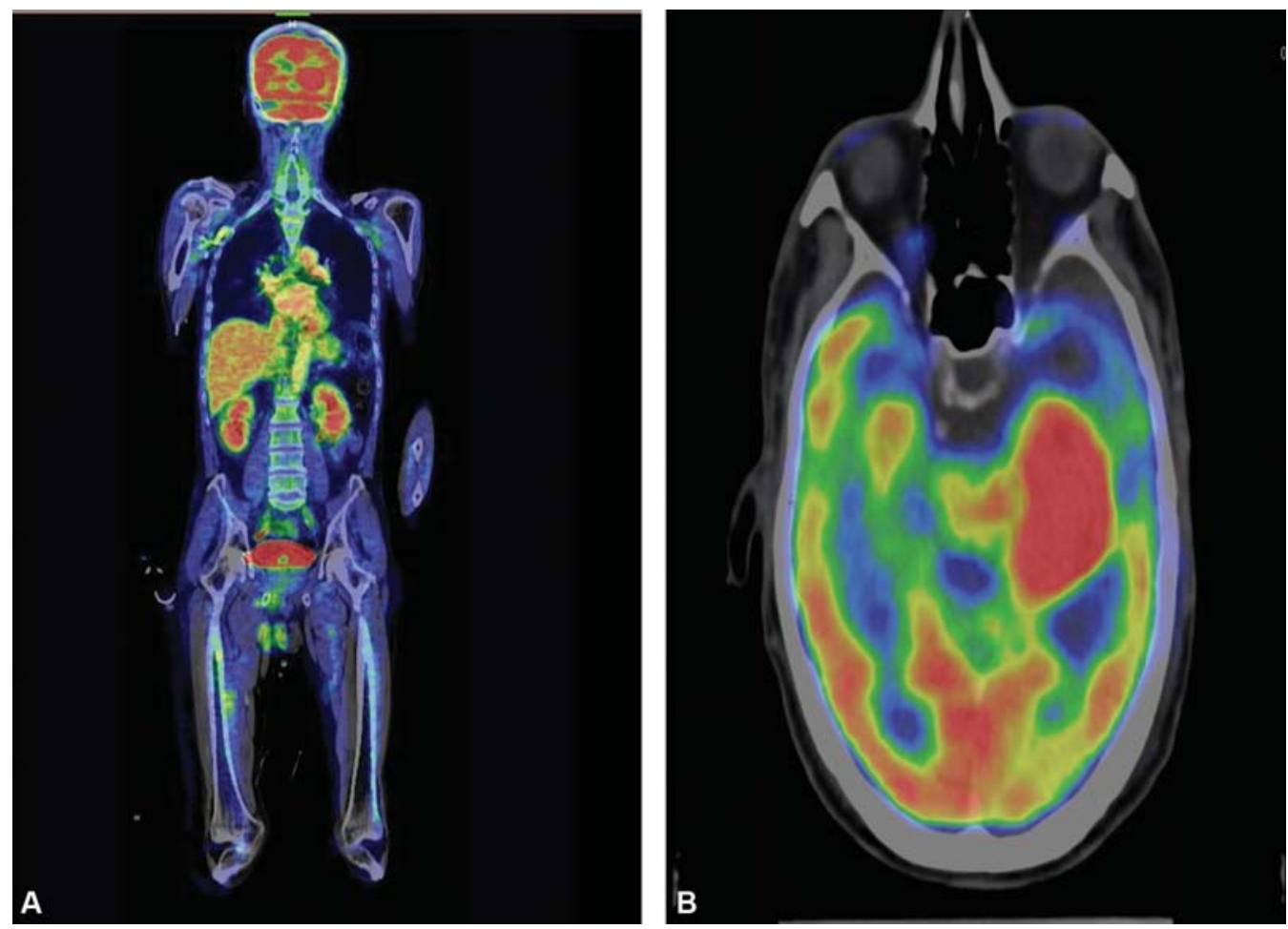

Fig. 4 (A, B) Whole body positron emission tomography computed tomography image showing no area of abnormal metabolic activity apart from focal hyperperfusion seen in the thalamocapsular region.

\section{Discussion}

In spite of a thorough evaluation by our renowned neuroradiological team and an elaborate discussion of the nature of lesion, a misdiagnosis could not be averted in our case necessitating consideration of fallacies of standard approach.

Primary CNS lymphoma is reported to be having an age standardized incidence of 0.4 to 0.5 per $1,00,000$ population per year, accounting for less than $1 \%$ of all non-Hodgkin's lymphomas and $3 \%$ of all brain malignancies. ${ }^{9-11}$ It has been documented to present with focal neurological deficits (70-73\%), neuropsychiatric symptoms (28-43\%), seizures (9-14\%), ocular symptoms (4\%), and increased intracranial pressure (3-33\%). ${ }^{12,13}$ Most lymphomas are supratentorial in an intraaxial location. The majority of supratentorial lesions occurs either in the cerebral white matter or within the corpus callosum, few in the deep central gray matter, in the posterior fossa, and rarely in the spinal cord. ${ }^{13}$ The majority of infratentorial tumors is found in the cerebellum. Seldom have there been any incidence of disease presenting both infra- and supratentorial. ${ }^{14}$ With respect to the IAM, it can present as an extra-axial mass, intra-axial mass extending into the cerebellopontine angle (CPA) or a leptomeningeal lymphoma presenting as a CPA mass. ${ }^{13}$

The first case report of a lymphoma presenting in bilateral IAC with symptoms of hearing loss and imbalance was only in 1993. ${ }^{15}$ Since then there have been a few publications similar to it. ${ }^{16,17}$ As per our knowledge, ours is the first report of a lymphoma that is unilateral and with uniqueness of involving only IAM and not brain parenchyma at presentation.

Various tumors of the IAM include VS, lipoma, facial neuroma, hemangioma, meningioma, neurofibroma, hamartoma, and arachnoidal cyst. MRI with contrast has time and again proved its efficacy as the investigation of choice for 


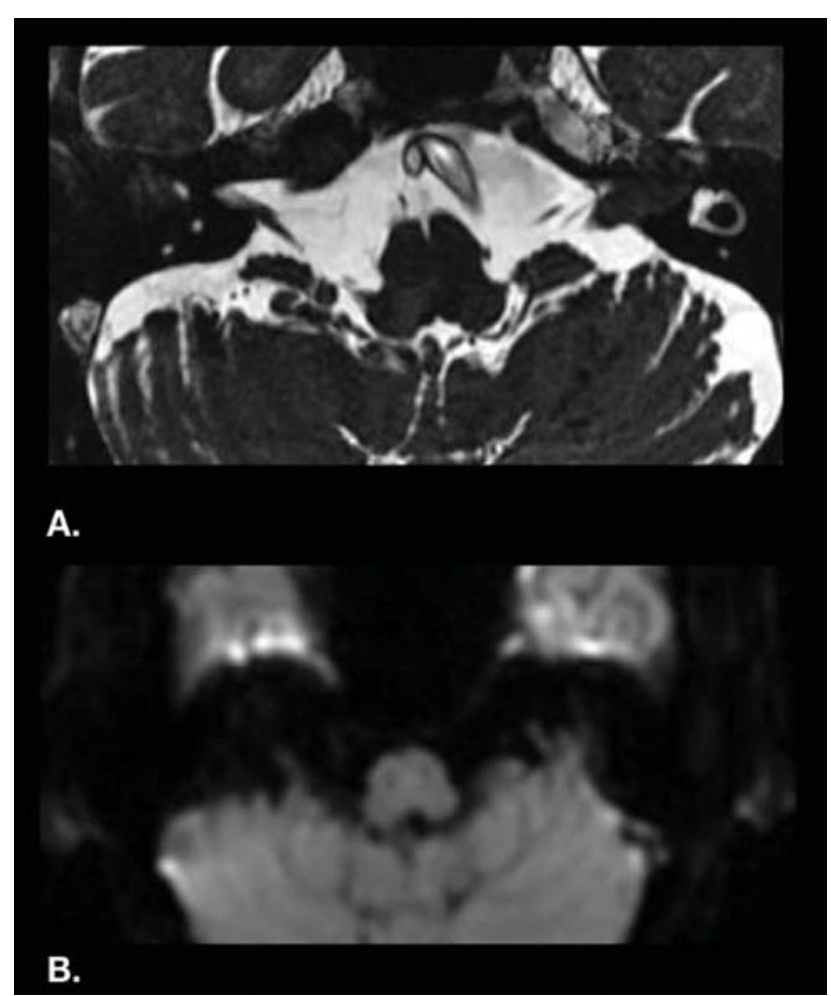

Fig. 5 (A) Axial FIESTA magnetic resonance imaging showing nodular $\mathrm{T} 2$ hypointense lesion in left cerebellopontine angle cistern extending into the internal acoustic meatus. (B) Diffusion-weighted imaging $(B=1,000)$ does not show any altered diffusivity or restricted diffusion.

lesions of the CPA/IAM. It has the advantage of being noninvasive, recognizing the site, extent, and peculiar signal intensities aiding in an apt diagnosis. ${ }^{18}$

VS-The majority of patients with a VS presents with asymmetrical hearing loss (slowly progressing) disequilibrium with or without asymmetrical tinnitus. ${ }^{19-21}$ In relation to the pons, schwannomas are usually isointense or mildly hypointense on T1-weighted images and mildly hyperintense on T2-weighted images. With relation to cerebrospinal fluid, it is noted to be hyperintense on $\mathrm{T} 1$ and isointense-hypointense on T2. ${ }^{22-26}$

Lipomas-They are thought to be congenital malformations arising from the primitive meninx that forms the subarachnoid space and meninges. ${ }^{27}$ The most common signs of CPA lipomas are hearing loss, tinnitus, vertigo, facial symptoms (facial and trigeminal nerve signs), and headaches..$^{28}$ When lipomas are viewed using $\mathrm{CT}$, they appear as hypodense masses ( -40 to -100 Hounsfield units [HU]) and do not enhance with contrast administration. ${ }^{29}$ On a T1-weighted MRI, lipomas are hyperintense, whereas on $\mathrm{T} 2$-weighted sequences, the appearance of a lipoma can vary from hypointense to hyperintense. Unique to lipomas is when fat suppression is applied, there is a decrease in or a complete loss of signal intensity from lipomas. ${ }^{29,30}$

Meningiomas-Meningiomas involving the IAM present either as tumors primarily originating in the IAM or those extending from adjacent regions. ${ }^{31,32} \mathrm{CT}$ scan shows characteristic calcification with or without adjacent bone hyperostosis or enostotic spurs. ${ }^{32}$ On MRI, they are isointense on T1 and T2, with homogeneous avid enhancement, similar to schwannomas. In 60 to $70 \%$ of cases, a "Dural tail" sign may be seen that represents enhancement of non-neoplastic thickening of the peritumoral dura. ${ }^{32}$

Cavernous hemangiomas of the IAM originate from the capillary bed of the epineurium surrounding Scarpa's ganglion and can either compress or infiltrate the nerve. ${ }^{11,33}$ Most common presentation is that of progressive hearing loss followed by facial nerve involvement ranging from facial spasm to facial paralysis of various grades. ${ }^{34}$ On MRI, the characteristic feature of cavernous hemangioma of the IAM is heterogeneous enhancement with gadolinium that distinguishes it from VS, while HRCT reveals irregular bony erosion of the IAM and characteristic intralesional calcification. ${ }^{35}$

Facial neuroma-Facial neuroma involving the IAM is a rare entity with patients presenting with facial palsy, paresis, or twitching that is unlikely with a VS. On MRI, lesion is mildly hypointense or isointense relative to brain on noncontrasted T1-weighted images and is enhanced following gadolinium administration. Heterogeneity or cystic change, well appreciated on T2-weighted images, may also be seen. ${ }^{36}$

In our case, we encountered IAM to be the primary site of presentation of a primary CNS lymphoma as the patient characteristically presented with unilateral sensorineural hearing loss and subjective giddiness. Cases of primary CNS lymphoma with tendency to spread perineurally have been known and reported in literature. ${ }^{11}$

\section{Conclusion}

To conclude, an intracanalicular lesion of IAM represents a VS in most instances. In the presence of facial paresis, this could possibly represent a facial neuroma, although they involve the labyrinthine/tympanic or mastoid segment of facial nerve. The above rare but possible differential diagnosis of lesions needs to be considered when dealing with the region reviewed carefully before concluding the lesion as VS only.

With improved imaging modalities, attention must be given to a detailed MRI. A thorough discussion with the neuroradiological team should be a routine protocol that could aid in avoiding potential misdiagnosis of the pathology.

\section{Author Contributions}

1. Operating surgeon, rafting the work or revising it critically for important intellectual content. Involved in final approval.

2. Substantial contributions to the conception or design of the work.

3. Radiology team involved in the process.

4. Radiology team involved in the process.

Conflict of Interest

- The authors have no ethical conflicts to disclose.

- The authors have no conflicts of interest to declare.

\section{References}

1 Howitz MF, Johansen C, Tos M, Charabi S, Olsen JH. Incidence of vestibular schwannoma in Denmark, 1977-1995. Am J Otol 2000; 21(05):690-694 
2 Lin D, Hegarty JL, Fischbein NJ, Jackler RK. The prevalence of "incidental" acoustic neuroma. Arch Otolaryngol Head Neck Surg 2005;131(03):241-244

3 Propp JM, McCarthy BJ, Davis FG, Preston-Martin S. Descriptive epidemiology of vestibular schwannomas. Neuro-oncol 2006;8 (01):1-11

4 Stangerup SE, Tos M, Thomsen J, Caye-Thomasen P. True incidence of vestibular schwannoma? Neurosurgery 2010;67(05):1335-1340 , discussion 1340

5 Parker GD, Harnsberger HR. Clinical-radiologic issues in perineural tumor spread of malignant diseases of the extracranial head and neck. Radiographics 1991;11(03):383-399

6 Brackmann DE, Bartels LJ. Rare tumors of the cerebellopontine angle. Otolaryngol Head Neck Surg (1979) 1980;88(05):555-559

7 Van der Meulen M, Dinmohamed AG, Visser O, Doorduijn JK, Bromberg JE. Improved survival in primary central nervous system lymphoma up to age 70 only: a population-based study on incidence, primary treatment and survival in the Netherlands, 1989-2015. Leukemia. 2017 Aug;31(8):1822.treatment and survival in the Netherlands, 1989-2015. Leukemia 2017;31:1822-1825

8 Villano JL, Koshy M, Shaikh H, Dolecek TA, McCarthy BJ. Age, gender, and racial differences in incidence and survival in primary CNS lymphoma. Br J Cancer 2011;105(09):1414-1418

9 Hoang-Xuan K, Bessell E, Bromberg J, et al;European Association for Neuro-Oncology Task Force on Primary CNS Lymphoma. Diagnosis and treatment of primary CNS lymphoma in immunocompetent patients: guidelines from the European Association for Neuro-Oncology. Lancet Oncol 2015;16(07):e322-e332

10 Lenarz M, Durisin M, Becker H, Lenarz T, Nejadkazem M. Primary central nervous system lymphoma presenting as bilateral tumors of the internal auditory canal. Skull Base 2007;17(06):409-412

11 Danchaivijitr N, Hesselink JR, Aryan HE, Herndier B. CerebelloPontine angle (CPA) lymphoma with perineural extension into the middle fossa: case report. Surg Neurol 2004;62(01):80-85

12 Coulon A, Lafitte F, Hoang-Xuan K, et al. Radiographic findings in 37 cases of primary CNS lymphoma in immunocompetent patients. Eur Radiol 2002;12(02):329-340

13 Garth RJN, Coddington R, Brightwell AP. Primary cerebral lymphoma presenting with bilateral cerebellopontine angle lesions. J Laryngol Otol 1993;107(10):937-939

14 Jaiswal AK, Mahapatra AK, Sharma MC. Primary central nervous lymphoma presenting as bilateral cerebellopontine angle lesions: a rare case report. J Clin Neurosci 2004;11(03):328-331

15 Larner AJ, D'Arrigo C, Scaravilli F, Howard RS. Bilateral symmetrical enhancing brainstem lesions: an unusual presentation of primary CNS lymphoma. Eur J Neurol 1999;6(06):721-723

16 Nishimura T, Uchida Y, Fukuoka M, Ono Y, Kurisaka M, Mori K. Cerebellopontine angle lymphoma: a case report and review of the literature. Surg Neurol 1998;50(05):480-485, discussion 485-486

17 Wang YT, Su HH, Hou Y, et al. Diffuse large B-cell lymphoma of the cerebellopontine angle in a patient with sudden hearing loss and facial palsy. Journal of the Chinese Medical Association. $2007 \mathrm{Jul}$ 1;70(7):294-7.... Wen CY, Huang PH, Chang CJ, Hsieh CT. Primary cerebellopontine angle lymphoma. Kaohsiung J Med Sci 2015;31 (01):55-56

18 Fortnum H, O'Neill C, Taylor $\mathrm{R}$, et al. The role of magnetic resonance imaging in the identification of suspected acoustic neuroma: a systematic review of clinical and cost effectiveness and natural history. Health Technol Assess 2009;13(18):iii-iv, ix$\mathrm{xi}, 1-154$

19 Godefroy WP, Kaptein AA, Vogel JJ, van der Mey AG. Conservative treatment of vestibular schwannoma: a follow-up study on clinical and quality-of-life outcome. Otol Neurotol 2009;30(07): 968-974

20 Press GA, Hesselink JR. MR imaging of cerebellopontine angle and internal auditory canal lesions at 1.5 T. AJR 1988;150(06): 1371-1381

21 Tali ET, Yuh WT, Nguyen HD, et al. Cystic acoustic schwannomas: MR characteristics. AJNR Am J Neuroradiol 1993;14(05): 1241-1247

22 Valvassori GE, Garcia Morales F, Palacios E, Dobben GE. MR of the normal and abnormal internal auditory canal. AJNR Am J Neuroradiol 1988;9(01):115-119

23 Daniels DL, Millen SJ, Meyer GA, et al. MR detection of tumor in the internal auditory canal. AJR Am J Roentgenol 1987;148(06): 1219-1222

24 Singh K, Singh MP, Thukral C, Rao K, Singh K, Singh A. Role of magnetic resonance imaging in evaluation of cerebellopontine angle schwannomas. Indian J Otolaryngol Head Neck Surg 2015; 67(01):21-27

25 Truwit CL, Barkovich AJ. Pathogenesis of intracranial lipoma: an MR study in 42 patients. AJNR Am J Neuroradiol 1990;11(04):665-674

26 Lagman C, Voth BL, Chung LK, et al. Evaluating the utility of a scoring system for lipomas of the cerebellopontine angle. Acta Neurochir (Wien) 2017;159(04):739-750

27 Scuotto A, Cappabianca S, D'Errico C, et al. Lipomas of the cerebellopontine angle: neuroradiological and surgical considerations. Review of the literature and report of our experience. Clin Neurol Neurosurg 2013;115(10):2280-2283

28 Bertot B, Steele WJ, Boghani Z, Britz G. Diagnostic dilemma: cerebellopontine angle lipoma versus dermoid cyst. Cureus 2017;9(11):e1894

29 von Eckardstein KL, Driscoll CL, Link MJ. Outcome after microsurgery for meningiomas involving the internal auditory canal. Neurosurgery 2010;67(05):1236-1242

30 Pollock BE, Link MJ, Foote RL, Stafford SL, Brown PD, Schomberg PJ. Radiosurgery as primary management for meningiomas extending into the internal auditory canal. Stereotact Funct Neurosurg 2004;82(2-3):98-103

31 Helie O, Soulie D, Sarrazin JL, Derosier C, Cordoliani YS, Cosnard G. [Magnetic resonance imaging and meningiomas of the posterior cerebral fossa. 31 cases]. J Neuroradiol 1995;22(04):252-270

32 Wallace EW. The dural tail sign. Radiology 2004;233(01):56-57

33 Barrera JE, Jenkins H, Said S. Cavernous hemangioma of the internal auditory canal: a case report and review of the literature. Am J Otolaryngol 2004;25(03):199-203

34 Omojola MF, al Hawashim NS, Zuwayed MA, al Ferayan A. CT and MRI features of cavernous haemangioma of internal auditory canal. Br J Radiol 1997;70(839):1184-1187

35 Shaida AM, McFerran DJ, da Cruz M, Hardy DG, Moffat DA. Cavernous haemangioma of the internal auditory canal. J Laryngol Otol 2000;114(06):453-455

36 Kertesz TR, Shelton C, Wiggins RH, Salzman KL, Glastonbury CM, Harnsberger R. Intratemporal facial nerve neuroma: anatomical location and radiological features. Laryngoscope 2001;111(07): $1250-1256$ 